Meta

Journal des traducteurs

Translators' Journal

\title{
Chantier terminologique
}

\section{Le bois hors forêt, étude de cas}

\section{Sofia Benyahia, France Brodeur et Monique Dion}

Volume 38, numéro 3, septembre 1993

URI : https://id.erudit.org/iderudit/002061ar

DOI : https://doi.org/10.7202/002061ar

Aller au sommaire du numéro

Éditeur(s)

Les Presses de l'Université de Montréal

ISSN

0026-0452 (imprimé)

1492-1421 (numérique)

Découvrir la revue

Citer cet article

Benyahia, S., Brodeur, F. \& Dion, M. (1993). Chantier terminologique : le bois hors forêt, étude de cas. Meta, 38(3), 517-527. https://doi.org/10.7202/002061ar d'utilisation que vous pouvez consulter en ligne.

https://apropos.erudit.org/fr/usagers/politique-dutilisation/ 


\section{CHANTIER TERMINOLOGIQUE Le bois hors forêt, étude de cas}

Étudiantes inscrites au séminaire de terminologie donné par André Clas à l'Université de Montréal, nous vous proposons de nous suivre dans la démarche terminologique que nous avons effectuée au trimestre d'hiver 93. Le chemin emprunté n'est pas nécessairement un modèle; il illustre simplement les décisions que nous avons prises pour en arriver à la création d' un arbre de domaine et d'une fiche terminologique informatisée. Enfin, la simplicité du sujet proposé est inversement proportionnelle à la complexité du processus mis en cuvre.

\section{INTRODUCTION}

Quel genre de démarche préside à l'élaboration d'un arbre de domaine, quelle orientation prendre, où élaguer, pourquoi ? Voilà un début plutôt confus qu'il a fallu débroussailler.

Le sujet proposé était le bois. Sujet vaste s'il en est! Cette «vastitude» représentait justement un attrait en terminologie pour apprendre à découper notre sujet, à en cerner les aspects qui nous intéressaient. De plus, le bois représentait, dans une certaine mesure, une notion dont nous n'avions pas à faire un long apprentissage. Au fil des semaines, nous nous sommes familiarisées avec notre sujet et notre attention s'est tout naturellement portée sur tout ce qui concernait le bois.

Nous avons d'abord recensé grossièrement tout ce qui touchait au bois, à une exception près : la transformation en pâte et papier. Cet aspect, qui embrasse un large éventail de notions, a été éliminé dès le départ. Cette décision visait à aiguiller notre recherche sur d'autres notions reliées au bois. Nous nous sommes vite rendu compte de la grande quantité d'ouvrages et d'articles de tous genres consacrés au domaine. La bibliothèque de l'Institut canadien de recherches en génie forestier contient environ 5000 documents, et son contenu a peu de chances de recouper celui de l'École québécoise du meuble et du bois ouvré qui renferme quelque mille ouvrages. Submergées par l'étendue de cette documentation, nous sommes sorties du bois... — c'est-à-dire de la forêt - en abordant le sujet sous l'angle de ses applications.

L'étude qui suit décrit par le menu les étapes suivies pour en arriver à l'élaboration d'un arbre de domaine et d'une fiche terminologique informatisée. Nous avons établi des critères précis quant à la sélection de la documentation bibliographique, la finalité autrement dit la commande d'un client fictif -, la disponibilité de la documentation et la rentabilité du travail. La terminologie, comme application pratique de la linguistique, doit fournir des résultats concrets dans des délais raisonnables et avec le maximum d'efficacité.

\section{RECHERCHE DOCUMENTAIRE ET EXPLORATION DU DOMAINE}

\section{Recherche documentaire}

En vue de faire l'inventaire des publications et des données liées au bois, nous avons d'abord recensé les organismes et les centres de documentation détenant de tels 
ouvrages et de tels renseignements. Les établissements où nous avons le plus abondamment puisé ont été les diverses bibliothèques de l'Université de Montréal (Lettres et sciences humaines, Aménagement, École polytechnique, etc.); l'Office de la langue française (bibliothèque et Banque de terminologie du Québec); le Secrétariat d'État du Canada (Bureau des traductions); l'École québécoise du meuble et du bois ouvré et l'Institut canadien de recherches en génie forestier. Nous avons de plus consulté des terminologues spécialistes du bois, parmi lesquels il faut mentionner Catherine Bowman et Reynald Binette.

À l'aide de ces diverses sources d'information, nous avons constitué une liste importante de documents portant sur notre sujet. Le caractère hétérogène de cette documentation est vite ressorti: un classement de celle-ci s'imposait donc. En l'examinant attentivement, nous en avons conclu qu'elle se répartissait en documentation primaire et secondaire. La documentation primaire regroupait des textes produits par des spécialistes du domaine, donc des manuels ou des articles traitant directement du bois: monographies, rapports, documents de centres de formation, manuels d'enseignement, articles d'encyclopédies et de revues spécialisées, actes de colloques, catalogues, index, etc., sans oublier les normes nationales et internationales. La documentation secondaire, quant à elle, réunissait les ouvrages et les travaux produits par des lexicographes ou des terminologues se basant sur la documentation primaire : dictionnaires, lexiques, glossaires et fiches terminologiques. Cette distinction nous est apparue importante, car la documentation primaire devait à notre avis constituer la principale source d'information.

Il est ensuite devenu évident que les nombreux documents recensés présentaient un intérêt très inégal pour la réalisation de notre projet, l'essentiel n'étant pas de disposer d'une documentation pléthorique, mais de pouvoir nous fonder sur des données fiables reflétant les connaissances partagées par les spécialistes du bois. Il fallait de ce fait éliminer un certain nombre de documents pour ne retenir que les plus pertinents. Une évaluation s'imposait alors. Les critères suivants ont été retenus :

a) fréquence de citation - ou «cote de fiabilité» — de l'auteur: on doit privilégier un auteur possédant une compétence et une renommée reconnues par ses pairs, celles-ci se traduisant tant par la richesse de production de cet auteur que par les mentions fréquentes de ses travaux dans les milieux spécialisés;

b) date de l'ouvrage: l'ouvrage le plus récent a la priorité, du moins dans le domaine technique; de la même manière, la dernière édition d'une publication prévaut;

c) critères méthodologiques suivis par l'auteur: on doit évidemment privilégier l'auteur faisant preuve de rigueur et de méthode dans sa démarche; la présence d'index est significative à cet égard;

d) références bibliographiques suffisantes dans l'ouvrage évalué: celles-ci sont une précieuse source d'information et leur présence confirme en partie la rigueur méthodologique dont fait preuve l'auteur; cela exclut toutefois les autocitations, c'est-à-dire les citations de l'auteur par lui-même;

e) version originale ou traduction: on doit dans la mesure du possible écarter les traductions; s'il faut les utiliser, ce sera avec circonspection.

Pour l'évaluation des dictionnaires, on peut ajouter les critères suivants :

f) qualité des articles: clarté des définitions, choix judicieux des contextes d'utilisation des termes, mention des sources;

g) renommée de l'ouvrage: une large diffusion et une consultation fréquente présupposent la qualité d'un ouvrage. 


\section{Exploration du domaine}

La meilleure façon d'avoir un bon aperçu des divers aspects liés au domaine du bois était d'examiner la nomenclature utilisée par divers spécialistes œuvrant dans ce secteur. Nous avons d'abord, à titre indicatif, étudié le classement utilisé dans le microfichier des bibliothèques de l'Université de Montréal (au mot-clef bois). Cette classification présente le désavantage d'être alphabétique, ce qui n'aide en rien dans la construction d'un arbre de domaine. Elle offre néanmoins un inventaire intéressant des divers éléments composant le domaine. Nous avons ensuite examiné la nomenclature utilisée par la Banque de terminologie du Québec (au mot-clef industrie du bois), puis celle de la Faculté des sciences forestières de l'Université Laval, et enfin les classifications d'organismes comme l'École québécoise du meuble et du bois ouvré et l'Institut canadien de recherches en génie forestier. Les ouvrages que nous avions retenus nous ont aussi fourni d'autres exemples de nomenclatures.

Au terme de cette collecte, en comparant ces divers modèles, nous avons pu observer que chaque organisme ou spécialiste avait élaboré sa propre nomenclature selon ses intérêts et ses besoins. Au moins deux leçons étaient à tirer de cette observation : d'une part, aucune classification n'est universelle, chacune étant ordonnée vers un but précis ; d'autre part, dans le cadre particulier de notre recherche, il devenait nécessaire de faire une critique et une synthèse des diverses nomenclatures recensées, pour construire notre propre arbre du domaine.

Dans l'élaboration d'un arbre en terminologie, ce sont des domaines et des sousdomaines que l'on classe. Au risque d'être accusées d'énoncer une lapalissade, nous avons dû conclure qu'un domaine est un ensemble de connaissances se subdivisant en sousdomaines: une structure arborescente, avec des branches et des ramilles. Cette définition minimaliste présente l'avantage de s'appliquer à toutes les démarches liées à l'organisation des connaissances.

Pour en revenir à l'exposé de notre démarche, l'examen comparatif des diverses nomenclatures recensées nous apprend que le classement du bois peut s'effectuer en se fondant sur trois critères principaux. On peut classer le bois en considérant les essences (résineux ou feuillus), ou encore les propriétés physiques et mécaniques du bois (densité, perméabilité, résistance, etc.), ou enfin ses utilisations finales (construction, fabrication de meubles, industrie chimique, etc.). Les diverses essences et les propriétés respectives de celles-ci entrent à notre avis dans un volet descriptif que nous intitulons généralités; celui-ci s'opposerait à un second grand volet regroupant toutes les utilisations du bois, que nous baptisons applications. Nous sommes donc arrivées dans un premier temps à l'esquisse d'un arbre comportant deux branches principales: généralités et applications. Mais il restait à voir si cette répartition résisterait à une étude plus poussée du domaine.

\section{LA CROISSANCE DE NOTRE ARBRE, DE LA PREMIÈRE À LA DERNIÈrE PROPOSITION}

\section{Premier arbre : usages et essences}

La division bicéphale généralités et applications nous gêne. Les nœuds terminologiques sont difficiles à poser à cause de l'étendue de chaque sous-domaine. Nous réexaminons les catégories relevées plus tôt et réorientons notre démarche.

Le thème qui nous guide dans notre première tentative d'élaboration d'arbre porte sur les usages du bois. Nos recherches nous ont amenées à constater que le bois avait des applications précises dans des secteurs déterminés. Nous dessinons donc un arbre sur les usages du bois dans trois secteurs, soit les usages chimiques, les usages artisanaux et les usages industriels. Le classement selon les usages nous permettra de nommer, tout au bas de l'arbre, les essences de bois qui sont affectées à telle ou telle application. 
Nous décidons de ne pas développer les usages chimiques, tels les vernis et les baumes, non plus que la pharmacologie à base d'extraits du bois. Sous chaque nœud nous voulons répartir les types de bois en fonction de leur utilisation, par exemple, la fabrication d'objets décoratifs ou la construction de bateaux. La division à laquelle nous pensons se fonde sur les bois précieux et non précieux. Pour le mobilier nous pourrions répartir les bois entre les tendres, les durs et les synthétiques. Notre premier arbre est un modèle bidirectionnel, ascendant ou descendant. Il nous permet de nous inspirer des usages pour en arriver aux types de bois ou des types de bois pour en arriver aux usages.

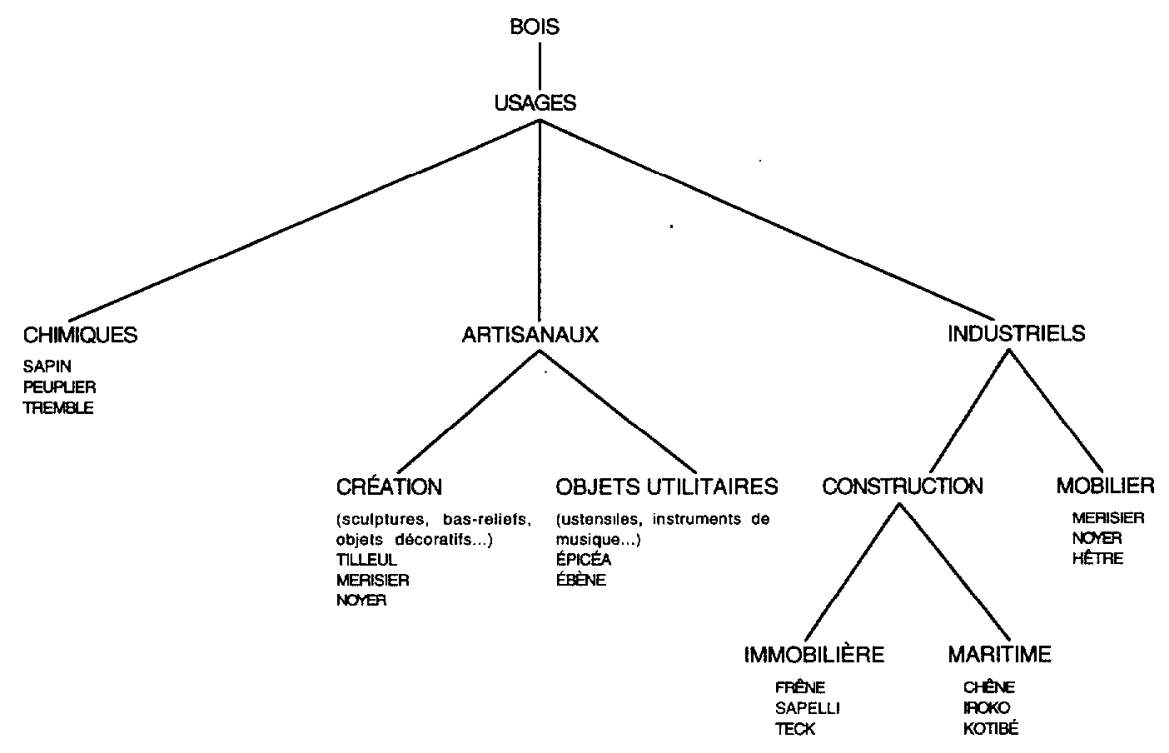

Arbre 1

La nomenclature des essences ne correspond cependant plus à notre finalité : le bois et ses usages. Nous délaissons les essences, car ce genre d'arbre de domaine s'apparente davantage au classement de la botanique. Nous revenons à la case départ; nous remettons en question la division même de notre arbre. Plus nous approfondissons le domaine, plus nous nous apercevons que nous le comprenons encore mal.

\section{Deuxième arbre: inversion de la démarche}

Nous faisons marche arrière, nous basant cette fois sur des ouvrages spécialisés. Nous serons influencées par les classements dans les trois ouvrages suivants: Éléments de physique du bois (Guillemette: 1982), Les bois du Canada (Mullins: 1981) et Le grand livre international du bois (adapt. Parmentier: 1977). Au fur et à mesure de l'élaboration de notre nouvel arbre, nous chercherons les «branchements» favorisant notre démarche. Autrement dit, pourquoi une étape nous conduit-elle à la suivante ? Nous nous rappelons sans cesse notre finalité : le bois et ses usages. Nous traitons du bois hors forêt, donc pas question de coupe, d'écorçage ou d'autres opérations réalisées en forêt. Notre intérêt pour le bois commence avec le transport de ce matériau vers les industries utilisatrices en fonction des applications finales. Nous divisons le bois en trois grandes catégories : bois à pâte, bois d'œuvre et bois à usages chimiques. 


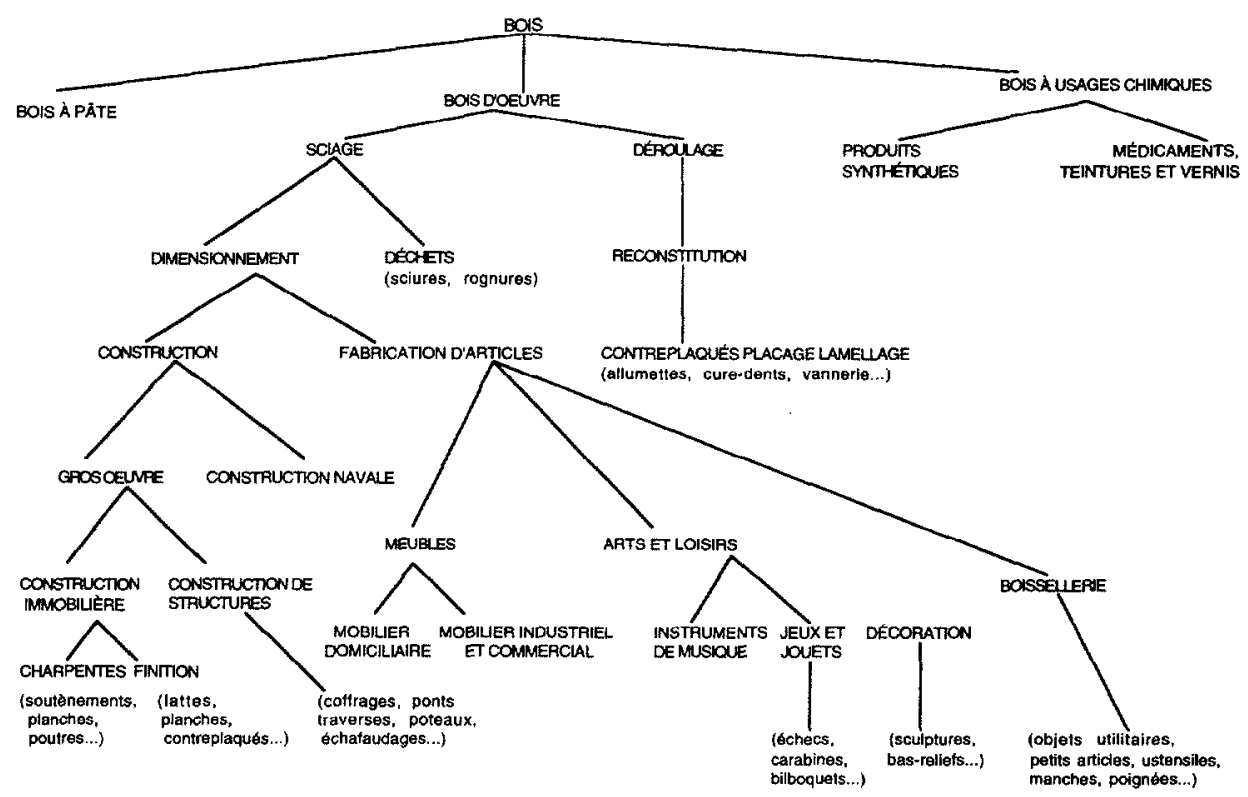

Arbre 2

Cet arbre nous semble mieux structuré, mais il ne nous convient pas encore. Nos trois grandes divisions nous satisfont, cependant nous craignons d'avoir oublié des procédés importants précédant les différents usages. Il nous apparaît important d'insérer le traitement du bois, sujet d'actualité au sein de l'industrie utilisatrice de ce matériau. Nous pensons aux divers types de bois traité que nous trouvons sur le marché, dont le bois étuvé, ignifugé et injecté de diverses substances comme le créosote. Dans notre arbre, le traitement devra également comprendre le non-traitement. Nous plaçons finalement l'étape du traitement après le sciage, mais constatons malgré tout que nous n'avons pas mentionné le séchage du bois, procédé qui, croyons-nous, devrait se situer avant le sciage. Nous réexaminons attentivement l'arbre.

\section{Troisième arbre : besoin d'équilibre et de synthèse}

Nous insérons donc le séchage et le traitement. Par souci de cohérence grammaticale, «dimensionnement» est remplacé par bois dimensionné. La notion de «bois reconstitué» est éliminée, cette expression n'étant probablement pas juste; nous l'avions créée pour préciser que le bois n'était pas scié, mais reconstitué sous une autre forme... Les usages médicinaux sont ajoutés dans la grande catégorie «bois à usages chimiques». La branche n'a pas été développée, mais il apparaît que cette spécialité doit y être indiquée. Lorsque des éléments sont ajoutés dans l'arbre, nous veillons à respecter la hiérarchie des sous-domaines. Selon nous, le sous-domaine «bois à pâte», que nous avons décidé depuis le début de ne pas développer, peut se diviser en plusieurs branches, de même pour le sous-domaine bois à usages chimiques et médicinaux. Par souci d'équilibre et de précision, nous mentionnons quelques exemples d'usages sous les deux sous-domaines. Ce faisant, nous voulons donner des pistes de recherche à la personne qui consulterait l'arbre. 


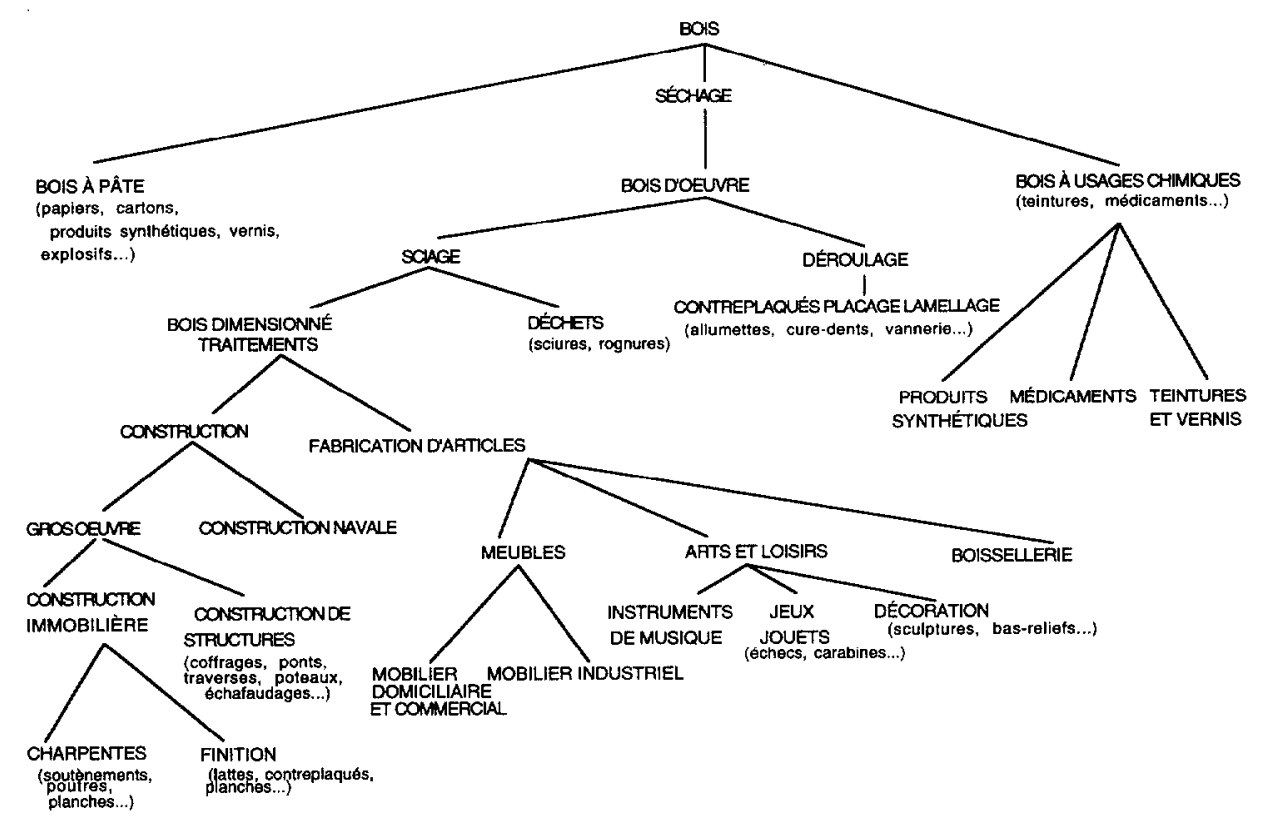

Arbre 3

Cet arbre se rapproche du but recherché, soit de présenter clairement les usages du bois. Cependant, les sous-domaines ajoutés ne font pas l'unanimité. En voulant être trop précises, nous avons inséré des imprécisions. En voulant équilibrer l'arbre, nous l'avons alourdi. Nous n'avons pas fait attention à la forme, notre attention étant fixée sur le fond. Nous n'avions pas encore remarqué qu'il y avait confusion entre procédés et produits.

\section{Arbre définitif : élagage et polissage}

C'est un émondage en règle que nous faisons subir à notre arbre! Le séchage nous apparaît tout à coup incongru. Nous avons inséré un procédé dans un arbre qui porte sur les usages. Le séchage est une opération qui aurait pu figurer dans un type d'arbre fondé sur les divers procédés que subit le bois. Diverses recherches et consultations nous amènent également à reclasser le traitement. Nous constatons en effet que la quasi-totalité des bois sont soumis à une forme quelconque de traitement, plus particulièrement les bois pour la construction de charpentes et de structures. La place du traitement dans notre arbre nous empêche de le détailler, à moins d'envisager l'élaboration d'un nouvel arbre sur les types de traitements selon les usages.

Nous en arrivons donc à un nouveau classement qui tient toujours compte de la cohérence grammaticale. Ainsi le bois d'œuvre sera subdivisé en bois traité, bois scié, bois amélioré et bois déroulé. Ce sont les quatre branches du bois d'œuvre qui occupent une place distincte dans l'industrie. Nous éliminons également les illustrations des sousdomaines qui nous ont servi pendant notre démarche. Afin de mieux situer les différentes ramifications du domaine, nous conservons tout de même les exemples d'usages sous les grandes catégories qui n'ont pas été développées. 


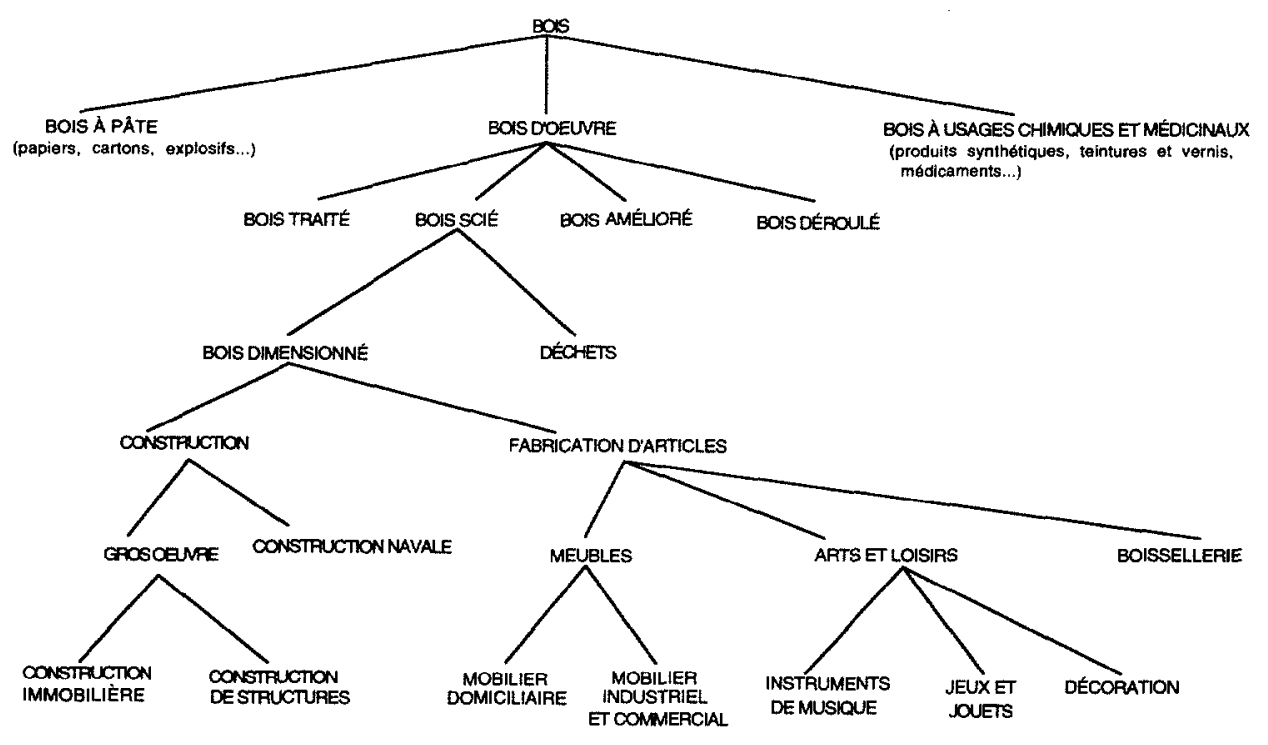

Arbre 4

\section{LA FICHE TERMINOLOGIQUE}

\section{Généralités}

La fiche terminologique est l'unité de base de l'information terminologique. Dans le cadre de cette recherche sur le bois, la fiche est bilingue anglais-français, et elle contient des renseignements variés répartis en douze champs, certains obligatoires et d'autres facultatifs comme l'indique le tableau suivant:

\begin{tabular}{|l|c|c|}
\hline & obligatoire & facultatif \\
\hline vedette & $\mathrm{X}$ & \\
\hline domaine & $\mathrm{X}$ & \\
\hline sous-domaine & $\mathrm{X}$ & \\
\hline catégorie gramm. & $\mathrm{X}$ & \\
\hline contexte & $\mathrm{X}$ & \\
\hline définition & & $\mathrm{X}$ \\
\hline synonyme & & $\mathrm{X}$ \\
\hline cooccurrence & & $\mathrm{X}$ \\
\hline source & $\mathrm{X}$ & \\
\hline remarque & & $\mathrm{X}$ \\
\hline identification du rédacteur & $\mathrm{X}$ & \\
\hline date de rédaction & $\mathrm{X}$ & \\
\hline
\end{tabular}

Deux sortes de règles ont présidé à l'élaboration de la fiche: des règles structurelles et des règles rédactionnelles. 


\section{Les règles structurelles}

Comme leur nom l'indique, les règles structurelles concernent la structure de la fiche informatisée. Elles sont appliquées au moment de la création de la base de données. Dans le logiciel utilisé (SGMB), les données sont classées par types; pour les besoins de notre recherche, nous en avons retenu quatre:

- le type TEXT fixe le nombre maximum de caractères que peut contenir un champ;

- le type NOTE est utilisé pour les champs dont la taille ne peut être déterminée à l'avance;

- le type DATE permet d'entrer la date de rédaction de la fiche conformément à la valeur implicite du système, c'est-à-dire mm-jj-aa pour 05-27-93, par exemple;

- le type INTEGER n'est utilisé que pour le champ pondération.

Le tableau qui suit représente la correspondance champ-type dans la fiche:

\begin{tabular}{|l|c|c|}
\hline champ & type & nombre de car. \\
\hline vedette & TEXT & 65 \\
\hline domaine & TEXT & 5 \\
\hline sous-domaine & TEXT & 20 \\
\hline catégorie gram. & NOTE & indéterminé \\
\hline contexte & NOTE & indéterminé \\
\hline définition & NOTE & indéterminé \\
\hline synonyme & NOTE & indéterminé \\
\hline cooccurrence & NOTE & indéterminé \\
\hline source & NOTE & indéterminé \\
\hline remarque & NOTE & indéterminé \\
\hline identification du rédacteur & TEXT & 3 \\
\hline date de rédaction & DATE & - \\
\hline pondération & INTEGER & - \\
\hline
\end{tabular}

\section{Les règles rédactionnelles}

Les règles rédactionnelles régissent quant à elles la présentation des données dans la fiche. Il s'agit d'une série de conventions typographiques établies pour uniformiser les productions et pour faciliter les échanges. Le développement qui suit spécifie l'application de ces règles dans différents champs de la fiche:

vedette Dans ce champ, les termes sont présentés sous forme lemmatisée, ou canonique.

\begin{tabular}{|c|c|}
\hline catégorie & lemmatisation \\
\hline nom & singulier \\
\hline verbe & infinitif \\
\hline adjectif & singulier \\
\hline adverbe & tel quel \\
\hline
\end{tabular}


Remarques :

1. Un terme est entré au singulier, mais s'il n'existe qu'au pluriel, il est enregistré au pluriel.

2. Les termes sont présentés en minuscules, sauf quand les majuscules sont obligatoires (noms propres, codes, sigles, etc.).

Exemples : comble à la Mansard triangulée; AFNOR.

3. Les variantes orthographiques sont consignées dans le champ remarque de la fiche. Exemples : contremarche, contre-marche.

domaine/sous-domaine

Un domaine et un sous-domaine sont attribués à chaque terme qui fait l'objet d'une entrée. L'écriture de ces champs est fonction du nombre de caractères permis par le type TEXT. Dans le cadre de la présente démarche, le domaine est bois dans tous les cas (d'où les 5 caractères); le ou les sous-domaines sont abrégés, le cas échéant, compte tenu des vingt caractères alloués à ce champ.

\section{catégorie grammaticale}

Ici se pose le problème du codage. Il s'agit d'assurer un maximum de clarté - le code doit être le plus mnémonique possible - et d'économie - éviter les frappes inutiles comme les points entre les lettres d'un sigle par exemple. Nous arrivons aux abréviations suivantes:

$\begin{array}{lll}\text { nom masculin } & \mathrm{nm} & \text { parquet } \\ \text { nom féminin } & \mathrm{nf} & \text { âme } \\ \text { nom masculin pluriel } & \mathrm{nmp} & - \\ \text { nom féminin pluriel } & \mathrm{nfp} & \text { dimensions tableau } \\ \text { nom masculin invariable } & \mathrm{nmi} & \text { hourdis } \\ \text { nom féminin invariable } & \mathrm{nfi} & - \\ \text { verbe transitif } & \mathrm{vt} & \text { équarrir } \\ \text { verbe intransitif } & \mathrm{vi} & - \\ \text { adjectif } & \mathrm{ad} & \text { poncé } \\ \text { adjectif invariable } & \text { adi } & \text { standard } \\ \text { adverbe } & \text { av } & -\end{array}$

\section{contexte}

Le type de contexte privilégié est celui qui contient le plus d'éléments révélateurs de la notion. Des signes linguistiques, comme «c'est-à-dire» ou «autrement dit», ou encore typographiques, comme les deux-points, facilitent le repérage d'un contexte de ce type.

Le contexte doit être ramené à l'essentiel, ainsi les passages inutiles peuvent être tronqués au moyen de points de suspension entre parenthèses. Les mots manquants sont ajoutés entre crochets pour éviter toute confusion possible.

\section{synonyme}

Chacun des synonymes fait l'objet d'une fiche. La mention voir $(X)$ en lieu et place du contexte renvoie au terme (X) de la fiche principale. Une fiche de renvoi est d'autant plus complète que la synonymie qu'elle représente est irrégulière. Le champ remarque sert dans certains cas à noter des différenciations non sémantiques entre des synonymes topolectes, chronolectes ou encore de fréquence d'utilisation variée. À titre d'exemple, l'épinette et l'épicéa désignent le même arbre, la première appellation étant utilisée au Canada, la seconde en France. 


\section{cooccurrence}

L'environnement syntaxique d'un terme est limité. Autrement dit, un terme s'emploie de préférence avec certains verbes, certains adjectifs ou autres types de mots. Ainsi, dans les sous-domaines de la construction ou de l'ameublement, un panneau de particules extrudé peut être plein ou évidé; on peut le scier, le découper, le clouer, le visser ou le plaquer (Dubuisson 1968: 793).

\section{source}

La source est donnée en clair par le nom de l'auteur, de la revue ou de la norme, selon les cas, suivi de la date de rédaction du document et de la page où l'information se trouve. La minuscule qui se trouve à la fin de certains champs constitue un appel de renvoi à la source.

Exemples : a) Binette 1984:85, b) Meta 1992:87

\section{pondération}

La pondération fait référence à l'usage des termes. Une échelle de 1 à 5 a été établie pour attribuer une valeur-indice à l'entrée :

1 terme normalisé

2 terme recommandé

3 usage courant

4 usage flottant

5 terme à éviter.

\section{Exemple de fiche}

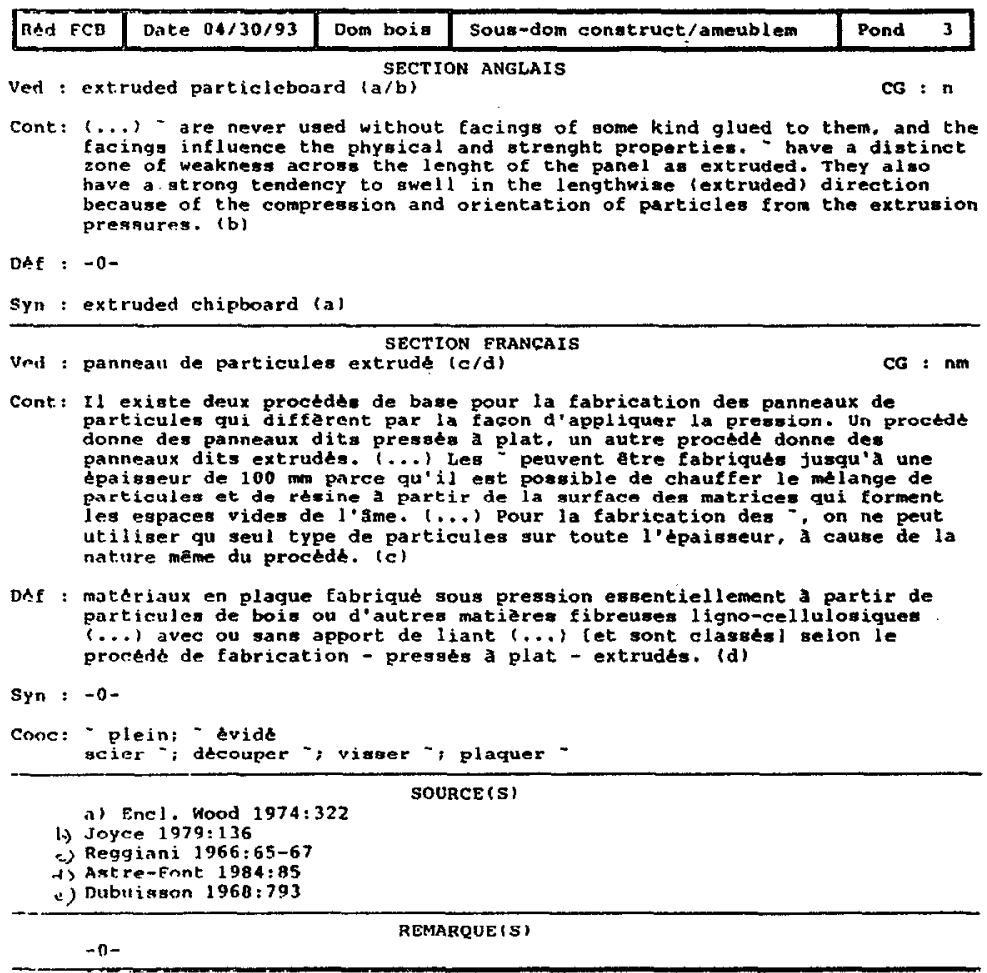




\section{CONCLUSION}

Les différentes étapes d'un travail terminographique se résument comme suit: 1) Délimiter le sujet de recherche ; 2) Respecter les critères de sélection de la documentation ; 3) Construire l'arbre du domaine en fonction d'un objectif précis ; 4) Élaborer la structure informatique de la fiche, et enfin 5) Consigner les données terminologiques dans cette fiche.

La voie empruntée n'est pas nécessairement un modèle; elle confirme simplement que tout travail de recherche, de synthèse et de présentation de résultats nécessite constance et ténacité de la part des terminologues.

Sofia Benyahia, France BRodeur, MoniQue Dion

\section{BIBLIOGRAPHIE}

La bibliographie présente un échantillon significatif des ouvrages généraux traitant du bois. Cependant, les ouvrages relevant des sous-domaines spécialisés mentionnés dans la fiche y sont indiqués.

ASTRE-FONT, M.-C. et al. (1984) : Précis d'ameublement : dessin, conception et normalisation, Paris, AFNOR, $201 \mathrm{p}$.

BOERHAVE BEEKMAN, W. (1964) : Elsevier's Wood Dictionary, New York, Elsevier (en 3 volumes).

CORKHILL, Thomas (1980) : The Complete Dictionary of Wood, New York, Stein and Day, 655 p.

DUBUISSON, Bernard (1968): Encyclopédie pratique de la construction et du bâtiment, Paris, Quillet (en 3 volumes).

FEIRER, John L. (1983): The Woodworker's Reference Guide and Sourcebook, New York, Charles Scribner's Sons, $353 \mathrm{p}$.

GUILLEMETTE, André (1982) : Éléments de physique du bois, cégep de Victoriaville et Direction générale des moyens d'enseignement du ministère de l'Éducation, Beauceville, Éditions FM, 153 p.

JACKSON, Albert et David DAY (1991): Le guide du bois, de la menuiserie et de l'ébénisterie, traduit par Aderald Van Luipen, Marie-Anne Tattevin et Paul Borie, Londres, La Maison rustique, $320 \mathrm{p}$.

JOYCE, Ernst (1979): The Encyclopedia of Furniture Making, New York, Sterling Publishing, $494 \mathrm{p}$.

Le grand livre international du bois (1977): adaptation française de Jean-Louis Parmentier (The International Book of Wood), Hong Kong, Fernand Nathan, 276 p.

MULLINS, E.J. et T.S. McKNIGHT (1981): Les bois du Canada : leurs propriétés et leurs usages, Montmagny, Éditions du Pélican en collaboration avec le Service canadien des forêts et le Centre d'édition du gouvernement du Canada, $509 \mathrm{p}$.

REGGIANI, J.-C. (1966) : Industries et commerce du bois, coll. Que sais-je ?, Paris, PUF, 128 p.

Terminologie de la sous-traitance : secteur du bois / Subcontracting Terminology: Wood Sector (1990): Bruxelles, Commission des communautés européennes, $864 \mathrm{p}$.

The Encyclopedia of Wood: Wood as an Engineering Material (1974): New York, Sterling, 376 p.

TRILlAT, H., B. AMPEAU et R.T. TRILLAT (1980, 1981, 1982): Technologie des métiers du bois, Paris, Dunod (en trois tomes).

\section{Revues spécialisées}

CTB Info, revue bimestrielle, Centre technique du bois, France.

Courrier de l'industriel du bois et de l'ameublement, revue trimestrielle, Centre technique du bois, France (ne paraît plus).

Le courrier du bois, revue trimestrielle, Bureau national de documentation sur le bois, Belgique.

L'industriel sur bois, revue mensuelle, Fédération romande des maîtres menuisiers, ébénistes, charpentiers, fabricants de meubles et parqueteurs, Suisse.

Revue du bois et de ses applicarions, mensuel, Société d'édition Soproge, France.

Wood / Le bois, revue trimestrielle, Canadian Wood Council, Canada.

Wood and Equipment News; Woodworking Materials, revue mensuelle, Westborn, Angleterre.

Wood and Wood Products, Vance Publishing, États-Unis (parution irrégulière).

Woodworking Industry, revue mensuelle, Benn Publications, Angleterre (ne paraît plus depuis juin 1991). 